\title{
Effective field theory for collective rotations and vibrations of triaxially deformed nuclei
}

\author{
Q. B. Chen, ${ }^{1}$ N. Kaiser, ${ }^{1}$ Ulf-G. Meißner, ${ }^{2,3}$ and J. Meng ${ }^{4,5,6}$ \\ ${ }^{1}$ Physik-Department, Technische Universität München, D-85747 Garching, Germany \\ ${ }^{2}$ Helmholtz-Institut für Strahlen- und Kernphysik and Bethe Center for Theoretical Physics, Universität Bonn, D-53115 Bonn, Germany \\ ${ }^{3}$ Institute for Advanced Simulation, Institut für Kernphysik, Jülich Center for Hadron Physics and JARA-HPC, \\ Forschungszentrum Jülich, D-52425 Jülich, Germany \\ ${ }^{4}$ State Key Laboratory of Nuclear Physics and Technology, School of Physics, Peking University, Beijing 100871, China \\ ${ }^{5}$ Yukawa Institute for Theoretical Physics, Kyoto University, Kyoto 606-8502, Japan \\ ${ }^{6}$ Department of Physics, University of Stellenbosch, Stellenbosch, South Africa
}

(Received 15 March 2018; published 26 June 2018)

\begin{abstract}
The effective field theory (EFT) for triaxially deformed even-even nuclei is generalized to include the vibrational degrees of freedom. The pertinent Hamiltonian is constructed up to next-to-leading order (NLO). The leadingorder part describes the vibrational motion, and the NLO part couples rotations to vibrations. The applicability of the EFT Hamiltonian is examined through the description of the energy spectra of the ground state bands, $\gamma$ bands, and $K=4$ bands in the ${ }^{108,110,112} \mathrm{Ru}$ isotopes. It is found that, by taking into account the vibrational degrees of freedom, the deviations for high-spin states in the $\gamma$ band observed in the EFT with only rotational degrees of freedom disappear. This supports the importance of including vibrational degrees of freedom in the EFT formulation for the collective motion of triaxially deformed nuclei.
\end{abstract}

DOI: 10.1103/PhysRevC.97.064320

\section{INTRODUCTION}

Effective field theory (EFT) is based on symmetry principles alone, and it exploits the separation of energy scales for the systematic construction of the Hamiltonian supplemented by a power counting. In this way, an increase in the number of parameters (i.e., low-energy constants that need to be adjusted to data) goes hand in hand with an increase in precision and thereby counterbalances the partial loss of predictive power. Actually, EFT often exhibits an impressive efficiency as highlighted by analytical results and economical means of calculations. In recent decades, EFT has enjoyed considerable successes in low-energy hadronic and nuclear structure physics. Pertinent examples include the descriptions of the nuclear interactions [1-3], halo nuclei [4-6], and nuclear fewbody systems [7-9]. Recently, Papenbrock and his collaborators have developed an EFT to describe the collective rotational and vibrational motions of deformed nuclei by [10-17].

Collective rotations and vibrations are the typical low-lying excitation modes of a nucleus. For a spherical nucleus, only vibrational modes exist. However, for a deformed nucleus, various vibrational bands are observed, and rotational bands are found to be built on the successive vibrational states [18,19]. Since the initial paper in 2011 [10], Papenbrock and his collaborators have completed a series of works devoted to the systematic treatment of nuclear collective motion in the EFT framework [11-17]. Through the application of EFT to deformed nuclei, the finer details of the experimental energy spectra, such as the change of the moment of inertia with spin, can be addressed properly through higher-order correction terms [10-14]. Moreover, in this approach the uncertainties of the theoretical model can be quantified [16], and a con- sistent treatment of electroweak currents together with the Hamiltonian can be obtained [15]. Let us note that all these investigations were restricted to axially symmetric nuclei.

Very recently, the pertinent EFT has been further generalized to describe the rotational motion of triaxially deformed even-even nuclei [20]. The triaxial deformation of a nucleus has been a subject of much interest in the study of nuclear structure for a long time. It is related to many interesting phenomena, including the $\gamma$ band [18], signature inversion [21], anomalous signature splitting [22], wobbling motion [18], chiral rotational modes [23], and most prominently multiple chiral doublet bands [24]. In fact, the wobbling motion and chiral doublet bands are regarded as unique fingerprints of stable triaxially deformed nuclei. In Ref. [20], the pertinent Hamiltonian has been constructed up to next-to-leading order (NLO). Taking the energy spectra of the ground state and $\gamma$ bands (together with some $K=4$ bands) in the ${ }^{102-112} \mathrm{Ru}$ isotopes as examples, the applicability of this novel EFT to triaxial nuclei was examined. It was found that the description at NLO is overall better than that at leading order (LO). Nevertheless, there were still some deviations between the NLO calculation and the data for some high-spin states in the $\gamma$ bands. The comparison to the results of a five-dimensional collective Hamiltonian (5DCH) based on covariant density functional theory (CDFT) [25] has indicated that the inclusion of vibrational degrees of freedom in the EFT formulation is important.

Therefore, in this paper, the vibrational degrees of freedom are additionally considered in the formulation of an EFT for collective nuclear motion. The pertinent Hamiltonian will be constructed up to NLO, where the LO part describes the vibrational motion, and the NLO part couples rotations to vibrations. The energy spectra of ground state bands, $\gamma$ bands, 
and $K=4$ bands in the ${ }^{108,110,112} \mathrm{Ru}$ isotopes are taken as examples to examine the applicability of our extended EFT approach.

The present paper is organized as follows. In Sec. II, the EFT for collective rotations and vibrations of triaxially deformed nuclei is constructed. The solutions of the rotational Hamiltonian in first-order perturbation theory are given in Sec. III. The obtained vibrational Hamiltonian is reduced in Sec. IV by expressing it in terms of the quadrupole deformation parameters $\beta_{2}$ and $\gamma_{2}$. The results of the corresponding quantum-mechanical calculations are presented and discussed in detail in Sec. V. Finally, a summary is given in Sec. VI together with perspectives for future research directions.

\section{CONSTRUCTION OF THE EFT}

In this section, the procedure of constructing the effective Lagrangian and Hamiltonian for collective rotations and vibrations is introduced and carried out. It follows similar steps as in the case of axially symmetric nuclei studied in Refs. [12,13] and in the case of collective rotations of triaxially deformed nuclei investigated in Ref. [20].

\section{A. Dynamical variables}

In an EFT with spontaneous symmetry breaking, the symmetry transformations are (typically) realized nonlinearly [26,27], and the Nambu-Goldstone fields parametrize the coset space $\mathcal{G} / \mathcal{H}$. Here, $\mathcal{G}$ is the symmetry group of the Hamiltonian and $\mathcal{H}$, the symmetry group of the ground state, is a proper subgroup of $\mathcal{G}$. The effective Lagrangian $\mathcal{L}$ is built from invariants that can be constructed from the fields in the coset space. In the following, we write the fields relevant for collective nuclear motion in the space-fixed coordinate frame, where the three generators of infinitesimal rotations about the space-fixed $x, y$, and $z$ axes are denoted by $J_{x}, J_{y}$, and $J_{z}$.

To describe a global rotation, the three Euler angles $\alpha, \beta$, and $\gamma$ serve as natural dynamical variables. On the classical level, they are purely time dependent and, upon quantization, they give rise to rotational bands. However, vibrations act locally on the nuclear surface and its location can be described by body-fixed spherical coordinates $r, \theta$, and $\phi$. Following the arguments in Refs. [12-14], one expects that Nambu-
Goldstone modes related to the radial coordinate $r$ have higher frequencies than those related to the angles $\theta$ and $\phi$. For low-energy excitations, one can therefore restrict the attention to the angular variables.

A triaxially deformed nucleus is invariant under the $180^{\circ}$ rotation about the body-fixed axes (discrete $\mathrm{D}_{2}$ symmetry), while the continuous $\mathrm{SO}(3)$ symmetry is broken by the deformation. Consequently, the Nambu-Goldstone modes lie in the three-dimensional coset space $\mathrm{SO}(3) / \mathrm{D}_{2}$. The modes depend on the angles $\theta$ and $\phi$ and the time $t$ and are generated from the nuclear ground state by a unitary transformation $U$. Following Refs. [12-14,20], the matrix $U$ can be parametrized in a product form as

$$
\begin{aligned}
U & =g(\alpha, \beta, \gamma) u(x, y, z), \\
g(\alpha, \beta, \gamma) & =\exp \left\{-i \alpha(t) J_{z}\right\} \exp \left\{-i \beta(t) J_{y}\right\} \exp \left\{-i \gamma(t) J_{z}\right\}, \\
u(x, y, z) & =\exp \left\{-i x(\theta, \phi, t) J_{x}-i y(\theta, \phi, t) J_{y}-i z(\theta, \phi, t) J_{z}\right\} .
\end{aligned}
$$

The fields $x(\theta, \phi, t), y(\theta, \phi, t)$, and $z(\theta, \phi, t)$ with $|x|,|y|,|z| \ll$ 1 generate small-amplitude vibrations of the nuclear surface. They depend on $\theta$ and $\phi$ such that their angular averages vanish:

$\int d \Omega x(\theta, \phi, t)=\int d \Omega y(\theta, \phi, t)=\int d \Omega z(\theta, \phi, t)=0$,

where $d \Omega=\sin \theta d \theta d \phi$ denotes the surface element on the unit sphere. Note that in the axially symmetric case $[12,13]$, $\gamma(t)$ and $z(\theta, \phi, t)$ do not exist as observable degrees of freedom, since the operator $J_{z}$ acting on the axially deformed ground state gives zero.

The power counting underlying the EFT is specified by [14]

$$
\begin{aligned}
\alpha, \beta, \gamma & \sim \mathcal{O}(1), \quad \dot{\alpha}, \dot{\beta}, \dot{\gamma} \sim \xi, \\
x, y, z & \sim \sqrt{\xi / \Omega}, \quad \dot{x}, \dot{y}, \dot{z} \sim \sqrt{\xi \Omega}, \quad \partial_{v} x, \partial_{\nu} y, \partial_{\nu} z \sim \sqrt{\xi / \Omega},
\end{aligned}
$$

where $\xi$ and $\Omega$ denote the energy scales of the rotational and vibrational motion, respectively. The dot refers to a time derivative and $\partial_{v}$ to angular derivatives with $v=\theta$ or $\phi$. Note that $\xi$ (typically $\approx 80 \mathrm{keV}$ ) is much smaller than $\Omega$ (typically $\approx 1 \mathrm{MeV}$ ), and hence $\sqrt{\xi / \Omega} \ll 1$ is a reasonably small parameter.

\section{B. Effective Lagrangian}

As usual, the effective Lagrangian is built from invariants. These are constructed from quantities $a_{\mu}^{x}$, $a_{\mu}^{y}$, and $a_{\mu}^{z}$ defined by $[12,13,20,26,27]$

$$
i U^{-1} \partial_{\mu} U=a_{\mu}^{x} J_{x}+a_{\mu}^{y} J_{y}+a_{\mu}^{z} J_{z}
$$

The symbol $\partial_{\mu}$ stands for a derivative with respect to one of the variables $\theta, \phi$, and $t$.

To work out the Nambu-Goldstone modes explicitly, we use the decompositions

$$
i g^{-1} \partial_{t} g=(-\dot{\alpha} \sin \beta \cos \gamma+\dot{\beta} \sin \gamma) J_{x}+(\dot{\alpha} \sin \beta \sin \gamma+\dot{\beta} \cos \gamma) J_{y}+(\dot{\alpha} \cos \beta+\dot{\gamma}) J_{z},
$$

and

$$
\begin{aligned}
i u^{-1} \partial_{t} u= & \left\{\dot{x}+\frac{1}{2}(\dot{y} z-y \dot{z})-\frac{1}{6}\left[\left(y^{2}+z^{2}\right) \dot{x}-x(y \dot{y}+z \dot{z})\right]\right\} J_{x}+\left\{\dot{y}+\frac{1}{2}(\dot{z} x-z \dot{x})-\frac{1}{6}\left[\left(x^{2}+z^{2}\right) \dot{y}-y(x \dot{x}+z \dot{z})\right]\right\} J_{y} \\
& +\left\{\dot{z}+\frac{1}{2}(\dot{x} y-x \dot{y})-\frac{1}{6}\left[\left(x^{2}+y^{2}\right) \dot{z}-z(x \dot{x}+y \dot{y})\right]\right\} J_{z}
\end{aligned}
$$


In the calculation of $i u^{-1} \partial_{t} u$, the expansion of $u$ in powers of $x, y$, and $z$ was performed up to cubic terms. With these formula, one obtains for the time derivative

$$
\begin{aligned}
i U^{-1} \partial_{t} U= & i u^{-1} \partial_{t} u+i u^{-1}\left(g^{-1} \partial_{t} g\right) u \\
= & \left\{\dot{x}+\frac{1}{2}(\dot{y} z-y \dot{z})-\frac{1}{6}\left[\left(y^{2}+z^{2}\right) \dot{x}-x(y \dot{y}+z \dot{z})\right]+(-\dot{\alpha} \sin \beta \cos \gamma+\dot{\beta} \sin \gamma)\right. \\
& +(\dot{\alpha} \sin \beta \sin \gamma+\dot{\beta} \cos \gamma) z-(\dot{\alpha} \cos \beta+\dot{\gamma}) y\} J_{x}+\left\{\dot{y}+\frac{1}{2}(\dot{z} x-z \dot{x})-\frac{1}{6}\left[\left(x^{2}+z^{2}\right) \dot{y}-y(x \dot{x}+z \dot{z})\right]\right. \\
& +(\dot{\alpha} \sin \beta \sin \gamma+\dot{\beta} \cos \gamma)+(\dot{\alpha} \cos \beta+\dot{\gamma}) x-(-\dot{\alpha} \sin \beta \cos \gamma+\dot{\beta} \sin \gamma) z\} J_{y}+\left\{\dot{z}+\frac{1}{2}(\dot{x} y-x \dot{y})\right. \\
& \left.-\frac{1}{6}\left[\left(x^{2}+y^{2}\right) \dot{z}-z(x \dot{x}+y \dot{y})\right]+(\dot{\alpha} \cos \beta+\dot{\gamma})+(-\dot{\alpha} \sin \beta \cos \gamma+\dot{\beta} \sin \gamma) y-(\dot{\alpha} \sin \beta \sin \gamma+\dot{\beta} \cos \gamma) x\right\} J_{z},
\end{aligned}
$$

while the angular derivatives $(v=\theta, \phi)$ read

$$
\begin{aligned}
i U^{-1} \partial_{\nu} U=i u^{-1} \partial_{\nu} u= & \left\{\partial_{\nu} x+\frac{1}{2}\left(z \partial_{\nu} y-y \partial_{\nu} z\right)-\frac{1}{6}\left[\left(y^{2}+z^{2}\right) \partial_{\nu} x-x\left(y \partial_{\nu} y+z \partial_{\nu} z\right)\right]\right\} J_{x} \\
& +\left\{\partial_{\nu} y+\frac{1}{2}\left(x \partial_{\nu} z-z \partial_{\nu} x\right)-\frac{1}{6}\left[\left(x^{2}+z^{2}\right) \partial_{\nu} y-y\left(x \partial_{\nu} x+z \partial_{\nu} z\right)\right]\right\} J_{y} \\
& +\left\{\partial_{\nu} z+\frac{1}{2}\left(y \partial_{\nu} x-x \partial_{\nu} y\right)-\frac{1}{6}\left[\left(x^{2}+y^{2}\right) \partial_{\nu} z-z\left(x \partial_{\nu} x+y \partial_{\nu} y\right)\right]\right\} J_{z} .
\end{aligned}
$$

In the expansions of $i U^{-1} \partial_{t} U$ in Eq. (8) we go up to order $\xi \sqrt{\xi / \Omega}$ to include the coupling terms between rotations and vibrations. Therefore, the quantities $u^{-1} J_{x} u, u^{-1} J_{y} u$, and $u^{-1} J_{z} u$ need to be expanded only up to order $\sqrt{\xi / \Omega}$, since $g^{-1} \partial_{t} g$ is of order $\xi$.

The components of the angular velocities follow from Eqs. (5) and (8) as

$$
\begin{aligned}
a_{t}^{x}= & \dot{x}+\left[\frac{1}{2}(\dot{y} z-y \dot{z})+(-\dot{\alpha} \sin \beta \cos \gamma+\dot{\beta} \sin \gamma)\right]+\left\{-\frac{1}{6}\left[\left(y^{2}+z^{2}\right) \dot{x}-x(y \dot{y}+z \dot{z})\right]\right. \\
& +(\dot{\alpha} \sin \beta \sin \gamma+\dot{\beta} \cos \gamma) z-(\dot{\alpha} \cos \beta+\dot{\gamma}) y\}, \\
a_{t}^{y}= & \dot{y}+\left[\frac{1}{2}(\dot{z} x-z \dot{x})+(\dot{\alpha} \sin \beta \sin \gamma+\dot{\beta} \cos \gamma)\right]+\left\{-\frac{1}{6}\left[\left(x^{2}+z^{2}\right) \dot{y}-y(x \dot{x}+z \dot{z})\right]\right. \\
& +(\dot{\alpha} \cos \beta+\dot{\gamma}) x-(-\dot{\alpha} \sin \beta \cos \gamma+\dot{\beta} \sin \gamma) z\}, \\
a_{t}^{z}= & \dot{z}+\left[\frac{1}{2}(\dot{x} y-x \dot{y})+(\dot{\alpha} \cos \beta+\dot{\gamma})\right]+\left\{-\frac{1}{6}\left[\left(x^{2}+y^{2}\right) \dot{z}-z(x \dot{x}+y \dot{y})\right]\right. \\
& +(-\dot{\alpha} \sin \beta \cos \gamma+\dot{\beta} \sin \gamma) y-(\dot{\alpha} \sin \beta \sin \gamma+\dot{\beta} \cos \gamma) x\},
\end{aligned}
$$

where the leading terms $(\dot{x}, \dot{y}$, and $\dot{z})$ are of order $\sqrt{\xi \Omega}$. The terms in square brackets are proportional to the energy scale $\xi$, and those in curly brackets scale as $\xi \sqrt{\xi / \Omega}$. Thus, successive terms in $a_{t}^{x, y, z}$ are suppressed by a relative factor $\sqrt{\xi / \Omega}$.

Moreover, one obtains from Eqs. (5) and (9) for the components involving angular derivatives

$$
\begin{aligned}
& a_{v}^{x}=\partial_{\nu} x+\frac{1}{2}\left(z \partial_{\nu} y-y \partial_{\nu} z\right)-\frac{1}{6}\left[\left(y^{2}+z^{2}\right) \partial_{\nu} x-x\left(y \partial_{\nu} y+z \partial_{\nu} z\right)\right], \\
& a_{\nu}^{y}=\partial_{\nu} y+\frac{1}{2}\left(x \partial_{\nu} z-z \partial_{\nu} x\right)-\frac{1}{6}\left[\left(x^{2}+z^{2}\right) \partial_{\nu} y-y\left(x \partial_{\nu} x+z \partial_{\nu} z\right)\right], \\
& a_{\nu}^{z}=\partial_{\nu} z+\frac{1}{2}\left(y \partial_{\nu} x-x \partial_{\nu} y\right)-\frac{1}{6}\left[\left(x^{2}+y^{2}\right) \partial_{\nu} z-z\left(x \partial_{\nu} x+y \partial_{\nu} y\right)\right],
\end{aligned}
$$

where the leading terms $\left(\partial_{\nu} x, \partial_{\nu} y\right.$, and $\left.\partial_{\nu} z\right)$ scale as $\sqrt{\xi / \Omega}$, the terms in parentheses as $\xi / \Omega$, and those in square brackets as $(\xi / \Omega)^{3 / 2}$. Again, successive terms in $a_{v}^{x, y, z}$ are suppressed by a relative factor $\sqrt{\xi / \Omega}$.

The expressions in Eqs. (10)-(15) give the lowest-order contributions from the Nambu-Goldstone modes $x, y, z$ and $\alpha, \beta, \gamma$ introduced in Eq. (1). Note that if $\gamma=0$ and $z=0$, then one recovers the axially symmetric case [12,13].

Respecting time-reversal invariance and the discrete $\mathrm{D}_{2}$ symmetry of a triaxial nucleus, ${ }^{1}$ only the squares of $a_{\mu}^{x}, a_{\mu}^{y}$, and $a_{\mu}^{z}$ may be used to construct the Lagrangian. For the temporal parts, one gets

$$
\begin{aligned}
\left(a_{t}^{x}\right)^{2}= & (-\dot{\alpha} \sin \beta \cos \gamma+\dot{\beta} \sin \gamma)^{2}+\dot{x}^{2}+(\dot{y} z-y \dot{z})(-\dot{\alpha} \sin \beta \cos \gamma+\dot{\beta} \sin \gamma)+2(\dot{\alpha} \sin \beta \sin \gamma+\dot{\beta} \cos \gamma) \dot{x} z \\
& -2(\dot{\alpha} \cos \beta+\dot{\gamma}) \dot{x} y+\frac{1}{4}(\dot{y} z-y \dot{z})^{2}-\frac{1}{3}\left[\left(y^{2}+z^{2}\right) \dot{x}^{2}-x \dot{x}(y \dot{y}+z \dot{z})\right], \\
\left(a_{t}^{y}\right)^{2}= & (\dot{\alpha} \sin \beta \sin \gamma+\dot{\beta} \cos \gamma)^{2}+\dot{y}^{2}+(\dot{z} x-z \dot{x})(\dot{\alpha} \sin \beta \sin \gamma+\dot{\beta} \cos \gamma)+2(\dot{\alpha} \cos \beta+\dot{\gamma}) \dot{y} x \\
& -2(-\dot{\alpha} \sin \beta \cos \gamma+\dot{\beta} \sin \gamma) \dot{y} z+\frac{1}{4}(\dot{z} x-z \dot{x})^{2}-\frac{1}{3}\left[\left(x^{2}+z^{2}\right) \dot{y}^{2}-y \dot{y}(x \dot{x}+z \dot{z})\right], \\
\left(a_{t}^{z}\right)^{2}= & (\dot{\alpha} \cos \beta+\dot{\gamma})^{2}+\dot{z}^{2}+(\dot{x} y-x \dot{y})(\dot{\alpha} \cos \beta+\dot{\gamma})+2(-\dot{\alpha} \sin \beta \cos \gamma+\dot{\beta} \sin \gamma) \dot{z} y-2(\dot{\alpha} \sin \beta \sin \gamma+\dot{\beta} \cos \gamma) \dot{z} x \\
& +\frac{1}{4}(\dot{x} y-x \dot{y})^{2}-\frac{1}{3}\left[\left(x^{2}+y^{2}\right) \dot{z}^{2}-z \dot{z}(x \dot{x}+y \dot{y})\right],
\end{aligned}
$$

\footnotetext{
${ }^{1}$ Under the four elements of $\mathrm{D}_{2}=Z_{2} \times Z_{2}$ the angular velocity vector $\left(a_{t}^{x}, a_{t}^{y}, a_{t}^{z}\right)$ is transformed into $\left(a_{t}^{x}, a_{t}^{y}, a_{t}^{z}\right),\left(a_{t}^{x},-a_{t}^{y},-a_{t}^{z}\right),\left(-a_{t}^{x}, a_{t}^{y},-a_{t}^{z}\right)$, and $\left(-a_{t}^{x},-a_{t}^{y}, a_{t}^{z}\right)$, respectively.
} 
where terms of order $\xi \Omega(\xi / \Omega)^{3 / 2}$ and higher have been consistently dropped. The invariants in Eqs. (16)-(18) can still be decomposed according to the power of the vibrational fields into

$$
\begin{aligned}
& \mathcal{L}_{1 a}=(-\dot{\alpha} \sin \beta \cos \gamma+\dot{\beta} \sin \gamma)^{2}, \\
& \mathcal{L}_{2 a}=\dot{x}^{2}+(\dot{y} z-y \dot{z})(-\dot{\alpha} \sin \beta \cos \gamma+\dot{\beta} \sin \gamma)+2(\dot{\alpha} \sin \beta \sin \gamma+\dot{\beta} \cos \gamma) \dot{x} z-2(\dot{\alpha} \cos \beta+\dot{\gamma}) \dot{x} y, \\
& \mathcal{L}_{3 a}=\frac{1}{4}(\dot{y} z-y \dot{z})^{2}-\frac{1}{3}\left[\left(y^{2}+z^{2}\right) \dot{x}^{2}-x \dot{x}(y \dot{y}+z \dot{z})\right], \\
& \mathcal{L}_{1 b}=(\dot{\alpha} \sin \beta \sin \gamma+\dot{\beta} \cos \gamma)^{2}, \\
& \mathcal{L}_{2 b}=\dot{y}^{2}+(\dot{z} x-z \dot{x})(\dot{\alpha} \sin \beta \sin \gamma+\dot{\beta} \cos \gamma)+2(\dot{\alpha} \cos \beta+\dot{\gamma}) \dot{y} x-2(-\dot{\alpha} \sin \beta \cos \gamma+\dot{\beta} \sin \gamma) \dot{y} z, \\
& \mathcal{L}_{3 b}=\frac{1}{4}(\dot{z} x-z \dot{x})^{2}-\frac{1}{3}\left[\left(x^{2}+z^{2}\right) \dot{y}^{2}-y \dot{y}(x \dot{x}+z \dot{z})\right], \\
& \mathcal{L}_{1 c}=(\dot{\alpha} \cos \beta+\dot{\gamma})^{2}, \\
& \mathcal{L}_{2 c}=\dot{z}^{2}+(\dot{x} y-x \dot{y})(\dot{\alpha} \cos \beta+\dot{\gamma})+2(-\dot{\alpha} \sin \beta \cos \gamma+\dot{\beta} \sin \gamma) \dot{z} y-2(\dot{\alpha} \sin \beta \sin \gamma+\dot{\beta} \cos \gamma) \dot{z} x, \\
& \mathcal{L}_{3 c}=\frac{1}{4}(\dot{x} y-x \dot{y})^{2}-\frac{1}{3}\left[\left(x^{2}+y^{2}\right) \dot{z}^{2}-z \dot{z}(x \dot{x}+y \dot{y})\right],
\end{aligned}
$$

where $\mathcal{L}_{1 a, 3 a}, \mathcal{L}_{1 b, 3 b}$, and $\mathcal{L}_{1 c, 3 c}$ are of order $\xi^{2}$. The leading terms $\left(\dot{x}^{2}, \dot{y}^{2}\right.$, and $\left.\dot{z}^{2}\right)$ in $\mathcal{L}_{2 a, 2 b, 2 c}$ are of order $\xi \Omega$ and the remaining terms scale as order $\xi^{2}$.

Next, we turn to the invariants constructed from derivatives with respect to the angles $\theta$ and $\phi$ listed in Eqs. (13)-(15). We restrict ourselves to terms of up to fourth order in $x, y, z$, and their derivatives. The pertinent squares read

$$
\begin{aligned}
& \left(a_{\nu}^{x}\right)^{2}=\left(\partial_{\nu} x\right)^{2}+\left[\left(\partial_{\nu} x\right)\left(z \partial_{\nu} y-y \partial_{\nu} z\right)\right]+\left\{\frac{1}{4}\left(z \partial_{\nu} y-y \partial_{\nu} z\right)^{2}-\frac{1}{3}\left(\partial_{\nu} x\right)\left[\left(y^{2}+z^{2}\right) \partial_{\nu} x-x\left(y \partial_{\nu} y+z \partial_{\nu} z\right)\right]\right\}, \\
& \left(a_{\nu}^{y}\right)^{2}=\left(\partial_{\nu} y\right)^{2}+\left[\left(\partial_{\nu} y\right)\left(x \partial_{\nu} z-z \partial_{\nu} x\right)\right]+\left\{\frac{1}{4}\left(x \partial_{\nu} z-z \partial_{\nu} x\right)^{2}-\frac{1}{3}\left(\partial_{\nu} y\right)\left[\left(x^{2}+z^{2}\right) \partial_{\nu} y-y\left(x \partial_{\nu} x+z \partial_{\nu} z\right)\right]\right\}, \\
& \left(a_{\nu}^{z}\right)^{2}=\left(\partial_{\nu} z\right)^{2}+\left[\left(\partial_{\nu} z\right)\left(y \partial_{\nu} x-x \partial_{\nu} y\right)\right]+\left\{\frac{1}{4}\left(y \partial_{\nu} x-x \partial_{\nu} y\right)^{2}-\frac{1}{3}\left(\partial_{\nu} z\right)\left[\left(x^{2}+y^{2}\right) \partial_{\nu} z-z\left(x \partial_{\nu} x+y \partial_{\nu} y\right)\right]\right\},
\end{aligned}
$$

where terms of order $(\xi / \Omega)^{5 / 2}$ and higher have been consistently dropped. In the above, $\left(a_{v}^{x}\right)^{2},\left(a_{v}^{y}\right)^{2}$, and $\left(a_{v}^{z}\right)^{2}$ are expressed in terms of $x, y$, and $z$, and their derivatives as polynomials of degree 2,3 , and 4 , respectively.

The partial derivatives $\partial_{\nu}$ can be replaced by the orbital angular momentum operator $\boldsymbol{L}$, whose components are

$$
\begin{aligned}
& L_{x}=i\left(\sin \phi \partial_{\theta}+\cot \theta \cos \phi \partial_{\phi}\right), \\
& L_{y}=i\left(-\cos \phi \partial_{\theta}+\cot \theta \sin \phi \partial_{\phi}\right), \\
& L_{z}=-i \partial_{\phi} .
\end{aligned}
$$

By reexpressing $\partial_{\theta}$ and $\partial_{\phi}$ in terms of $L_{x}, L_{y}$, and $L_{z}$, one constructs from the first terms in Eqs. (28)-(30) the following six Lagrangians:

$$
\begin{aligned}
& \mathcal{L}_{4 a}=(\boldsymbol{L} x)^{2}, \quad \mathcal{L}_{4 b}=(\boldsymbol{L} y)^{2}, \quad \mathcal{L}_{4 c}=(\boldsymbol{L} z)^{2}, \\
& \mathcal{L}_{4 d}=\left(L_{z} x\right)^{2}, \quad \mathcal{L}_{4 e}=\left(L_{z} y\right)^{2}, \quad \mathcal{L}_{4 f}=\left(L_{z} z\right)^{2} .
\end{aligned}
$$

In the same way, the second terms in square brackets lead to

$$
\begin{aligned}
\mathcal{L}_{5 a} & =(\boldsymbol{L} x)[z(\boldsymbol{L} y)-y(\boldsymbol{L} z)], \\
\mathcal{L}_{5 b} & =(\boldsymbol{L} y)[x(\boldsymbol{L} z)-z(\boldsymbol{L} x)], \\
\mathcal{L}_{5 c} & =(\boldsymbol{L} z)[y(\boldsymbol{L} x)-x(\boldsymbol{L} y)], \\
\mathcal{L}_{5 d} & =\left(L_{y} x\right)\left[z\left(L_{x} y\right)-y\left(L_{x} z\right)\right]+\left(L_{x} x\right)\left[z\left(L_{y} y\right)-y\left(L_{y} z\right)\right], \\
\mathcal{L}_{5 e} & =\left(L_{y} y\right)\left[x\left(L_{x} z\right)-z\left(L_{x} x\right)\right]+\left(L_{x} y\right)\left[x\left(L_{y} z\right)-z\left(L_{y} x\right)\right], \\
\mathcal{L}_{5 f} & =\left(L_{y} z\right)\left[y\left(L_{x} x\right)-x\left(L_{x} y\right)\right]+\left(L_{x} z\right)\left[y\left(L_{y} x\right)-x\left(L_{y} y\right)\right],
\end{aligned}
$$

and the third terms in curly brackets give rise to

$$
\begin{aligned}
\mathcal{L}_{6 a}= & \frac{1}{4}[z(\boldsymbol{L} y)-y(\boldsymbol{L} z)]^{2}+\frac{1}{3}(\boldsymbol{L} x)\left\{x[y(\boldsymbol{L} y)+z(\boldsymbol{L} z)]-\left(y^{2}+z^{2}\right)(\boldsymbol{L} x)\right\}, \\
\mathcal{L}_{6 b}= & \frac{1}{4}[x(\boldsymbol{L} z)-z(\boldsymbol{L} x)]^{2}+\frac{1}{3}(\boldsymbol{L} y)\left\{y[z(\boldsymbol{L} z)+x(\boldsymbol{L} x)]-\left(z^{2}+x^{2}\right)(\boldsymbol{L} y)\right\}, \\
\mathcal{L}_{6 c}= & \frac{1}{4}[y(\boldsymbol{L} x)-x(\boldsymbol{L} y)]^{2}+\frac{1}{3}(\boldsymbol{L} z)\left\{z[x(\boldsymbol{L} x)+y(\boldsymbol{L} y)]-\left(x^{2}+y^{2}\right)(\boldsymbol{L} z)\right\}, \\
\mathcal{L}_{6 d}= & \frac{1}{2}\left[z\left(L_{y} y\right)-y\left(L_{y} z\right)\right]\left[z\left(L_{x} y\right)-y\left(L_{x} z\right)\right]+\frac{1}{3} x\left\{\left(L_{y} x\right)\left[y\left(L_{x} y\right)+z\left(L_{x} z\right)\right]+\left(L_{x} x\right)\left[y\left(L_{y} y\right)+z\left(L_{y} z\right)\right]\right\} \\
& -\frac{2}{3}\left(y^{2}+z^{2}\right)\left(L_{x} x\right)\left(L_{y} x\right),
\end{aligned}
$$




$$
\begin{aligned}
\mathcal{L}_{6 e}= & \frac{1}{2}\left[x\left(L_{y} z\right)-z\left(L_{y} x\right)\right]\left[x\left(L_{x} z\right)-z\left(L_{x} x\right)\right]+\frac{1}{3} y\left\{\left(L_{y} y\right)\left[z\left(L_{x} z\right)+x\left(L_{x} x\right)\right]+\left(L_{x} y\right)\left[z\left(L_{y} z\right)+x\left(L_{y} x\right)\right]\right\} \\
& -\frac{2}{3}\left(z^{2}+x^{2}\right)\left(L_{x} y\right)\left(L_{y} y\right), \\
\mathcal{L}_{6 f}= & \frac{1}{2}\left[y\left(L_{y} x\right)-x\left(L_{y} y\right)\right]\left[y\left(L_{x} x\right)-x\left(L_{x} y\right)\right]+\frac{1}{3} z\left\{\left(L_{y} z\right)\left[x\left(L_{x} x\right)+y\left(L_{x} y\right)\right]+\left(L_{x} z\right)\left[x\left(L_{y} x\right)+y\left(L_{y} y\right)\right]\right\} \\
& -\frac{2}{3}\left(x^{2}+y^{2}\right)\left(L_{x} z\right)\left(L_{y} z\right) .
\end{aligned}
$$

As a result, the total effective Lagrangian $L$ is given by the angular average of an arbitrary linear combination of the invariants constructed above and it involves 27 low-energy constants (LECs)

$$
\begin{aligned}
L & =L_{1}+L_{2}+L_{3}+L_{4}+L_{5}+L_{6} \\
& =\frac{1}{4 \pi} \int d \Omega\left[\sum_{i=a, b, c}\left(\frac{A_{i}}{2} \mathcal{L}_{1 i}+\frac{B_{i}}{2} \mathcal{L}_{2 i}+\frac{C_{i}}{2} \mathcal{L}_{3 i}\right)-\sum_{i=a, b, c, d, e, f}\left(\frac{D_{i}}{2} \mathcal{L}_{4 i}+\frac{E_{i}}{2} \mathcal{L}_{5 i}+\frac{F_{i}}{2} \mathcal{L}_{6 i}\right)\right]
\end{aligned}
$$

Following Ref. [12], we expand the real function $x(\theta, \phi, t)$ in terms of the real orthonormal functions $Z_{\lambda \mu}$, called tesseral harmonics, as

$$
x(\theta, \phi, t)=\sum_{\lambda=2}^{\infty} \sum_{\mu=-\lambda}^{\lambda} x_{\lambda \mu}(\theta, \phi, t) Z_{\lambda \mu}(\theta, \phi),
$$

and in the same way for the real variables $y, z, \dot{x}, \dot{y}$, and $\dot{z}$. The tesseral harmonics $Z_{\lambda \mu}(\theta, \phi)$ are related to the spherical harmonics $Y_{\lambda \mu}(\theta, \phi)$ by

$$
Z_{\lambda \mu}= \begin{cases}\frac{1}{\sqrt{2}}\left(Y_{\lambda \mu}+Y_{\lambda \mu}^{*}\right), & \mu>0, \\ Y_{\lambda 0}, & \mu=0, \\ -\frac{i}{\sqrt{2}}\left(Y_{\lambda \mu}-Y_{\lambda \mu}^{*}\right), & \mu<0 .\end{cases}
$$

It is obvious that the expansion coefficients $x_{\lambda \mu}$ are real. Note that in Eq. (49) the contributions with $\lambda=0$ and $\lambda=1$ must be excluded, since $\lambda=0$ violates Eq. (2) (volume conservation), while $\lambda=1$ describes global translations in space.

Using these expansions and carrying out the solid angle integration, the total Lagrangian takes the form

$$
\begin{aligned}
L= & \frac{A_{a}}{2} \omega_{x}^{2}+\frac{A_{b}}{2} \omega_{y}^{2}+\frac{A_{c}}{2} \omega_{z}^{2} \\
& +\frac{B_{a}}{2} \sum_{\lambda \mu}\left[\dot{x}_{\lambda \mu}^{2}+\left(\dot{y}_{\lambda \mu} z_{\lambda \mu}-y_{\lambda \mu} \dot{z}_{\lambda \mu}\right) \omega_{x}+2 \omega_{y} \dot{x}_{\lambda \mu} z_{\lambda \mu}-2 \omega_{z} \dot{x}_{\lambda \mu} y_{\lambda \mu}\right] \\
& +\frac{B_{b}}{2} \sum_{\lambda \mu}\left[\dot{y}_{\lambda \mu}^{2}+\left(\dot{z}_{\lambda \mu} x_{\lambda \mu}-z_{\lambda \mu} \dot{x}_{\lambda \mu}\right) \omega_{y}+2 \omega_{z} \dot{y}_{\lambda \mu} x_{\lambda \mu}-2 \omega_{x} \dot{y}_{\lambda \mu} z_{\lambda \mu}\right] \\
& +\frac{B_{c}}{2} \sum_{\lambda \mu}\left[\dot{z}_{\lambda \mu}^{2}+\left(\dot{x}_{\lambda \mu} y_{\lambda \mu}-x_{\lambda \mu} \dot{y}_{\lambda \mu}\right) \omega_{z}+2 \omega_{x} \dot{z}_{\lambda \mu} y_{\lambda \mu}-2 \omega_{y} \dot{z}_{\lambda \mu} x_{\lambda \mu}\right] \\
& -\frac{1}{2} \sum_{\lambda \mu}\left\{\lambda(\lambda+1)\left[D_{a} x_{\lambda \mu}^{2}+D_{b} y_{\lambda \mu}^{2}+D_{c} z_{\lambda \mu}^{2}\right]+\mu^{2}\left[D_{d} x_{\lambda \mu}^{2}+D_{e} y_{\lambda \mu}^{2}+D_{f} z_{\lambda \mu}^{2}\right]\right\}
\end{aligned}
$$

where we restrict ourselves to terms up to orders with $\xi, \Omega$, and $\sqrt{\xi \Omega}$. In addition, we confine ourselves to harmonic vibrations of the nuclear surface and hence do not include the anharmonicity terms $\mathcal{L}_{3 i}$, similar as in Refs. [12,13]. In this way the number of LECs gets reduced to 12. The pure rotor part [first line in Eq. (51)] is written in terms of squares of

$$
\begin{aligned}
& \omega_{x}=-\dot{\alpha} \sin \beta \cos \gamma+\dot{\beta} \sin \gamma, \\
& \omega_{y}=\dot{\alpha} \sin \beta \sin \gamma+\dot{\beta} \cos \gamma, \\
& \omega_{z}=\dot{\alpha} \cos \beta+\dot{\gamma},
\end{aligned}
$$

which are the rotational frequencies (in the body-fixed frame) expressed through Euler angles and their time derivatives [20]. 
Finally, we study the dependence of the parameters on the energy scales. Since $\omega_{x, y, z} \sim \xi$, one requires $A_{a, b, c} \sim \xi^{-1}$ so that the rotational energy scales as order $\xi$. Similarly, $\dot{x}_{\lambda \mu}, \dot{y}_{\lambda \mu}$, and $\dot{z}_{\lambda \mu} \sim \sqrt{\xi \Omega}$ lead to $B_{a, b, c} \sim \xi^{-1}$ so that the vibrational energy scales as order $\Omega$. This implies that the rotation-vibration coupling term is of order $\xi^{-1} \sqrt{\xi \Omega} \sqrt{\xi / \Omega} \xi=\xi$. In addition, the scaling behavior $D_{a-f} \sim \Omega^{2} / \xi$ implies that the collective potential [last line in Eq. (51)] is of order $\Omega$.

\section{Canonical momenta}

From the Lagrangian (51), one derives the following canonical momenta:

$$
\begin{aligned}
& p_{\alpha}=\frac{\partial L}{\partial \dot{\alpha}}=-\sin \beta \cos \gamma\left[A_{a} \omega_{x}+\frac{B_{a}}{2} \sum_{\lambda \mu}\left(\dot{y}_{\lambda \mu} z_{\lambda \mu}-y_{\lambda \mu} \dot{z}_{\lambda \mu}\right)-B_{b} \sum_{\lambda \mu} \dot{y}_{\lambda \mu} z_{\lambda \mu}+B_{c} \sum_{\lambda \mu} \dot{z}_{\lambda \mu} y_{\lambda \mu}\right] \\
& +\sin \beta \sin \gamma\left[A_{b} \omega_{y}+B_{a} \sum_{\lambda \mu} \dot{x}_{\lambda \mu} z_{\lambda \mu}+\frac{B_{b}}{2} \sum_{\lambda \mu}\left(\dot{z}_{\lambda \mu} x_{\lambda \mu}-z_{\lambda \mu} \dot{x}_{\lambda \mu}\right)-B_{c} \sum_{\lambda \mu} \dot{z}_{\lambda \mu} x_{\lambda \mu}\right] \\
& +\cos \beta\left[A_{c} \omega_{z}-B_{a} \sum_{\lambda \mu} \dot{x}_{\lambda \mu} y_{\lambda \mu}+B_{b} \sum_{\lambda \mu} \dot{y}_{\lambda \mu} x_{\lambda \mu}+\frac{B_{c}}{2} \sum_{\lambda \mu}\left(\dot{x}_{\lambda \mu} y_{\lambda \mu}-x_{\lambda \mu} \dot{y}_{\lambda \mu}\right)\right] \\
& p_{\beta}=\frac{\partial L}{\partial \dot{\beta}}=\sin \gamma\left[A_{a} \omega_{x}+\frac{B_{a}}{2} \sum_{\lambda \mu}\left(\dot{y}_{\lambda \mu} z_{\lambda \mu}-y_{\lambda \mu} \dot{z}_{\lambda \mu}\right)-B_{b} \sum_{\lambda \mu} \dot{y}_{\lambda \mu} z_{\lambda \mu}+B_{c} \sum_{\lambda \mu} \dot{z}_{\lambda \mu} y_{\lambda \mu}\right] \\
& +\cos \gamma\left[A_{b} \omega_{y}+B_{a} \sum_{\lambda \mu} \dot{x}_{\lambda \mu} z_{\lambda \mu}+\frac{B_{b}}{2} \sum_{\lambda \mu}\left(\dot{z}_{\lambda \mu} x_{\lambda \mu}-z_{\lambda \mu} \dot{x}_{\lambda \mu}\right)-B_{c} \sum_{\lambda \mu} \dot{z}_{\lambda \mu} x_{\lambda \mu}\right], \\
& p_{\gamma}=\frac{\partial L}{\partial \dot{\gamma}}=A_{c} \omega_{z}-B_{a} \sum_{\lambda \mu} \dot{x}_{\lambda \mu} y_{\lambda \mu}+B_{b} \sum_{\lambda \mu} \dot{y}_{\lambda \mu} x_{\lambda \mu}+\frac{B_{c}}{2} \sum_{\lambda \mu}\left(\dot{x}_{\lambda \mu} y_{\lambda \mu}-x_{\lambda \mu} \dot{y}_{\lambda \mu}\right), \\
& p_{\lambda \mu}^{x}=\frac{\partial L}{\partial \dot{x}_{\lambda \mu}}=B_{a}\left[\dot{x}_{\lambda \mu}+\omega_{y} z_{\lambda \mu}-\omega_{z} y_{\lambda \mu}\right]-\frac{B_{b}}{2} z_{\lambda \mu} \omega_{y}+\frac{B_{c}}{2} y_{\lambda \mu} \omega_{z}, \\
& p_{\lambda \mu}^{y}=\frac{\partial L}{\partial \dot{y}_{\lambda \mu}}=\frac{B_{a}}{2} z_{\lambda \mu} \omega_{x}+B_{b}\left[\dot{y}_{\lambda \mu}+\omega_{z} x_{\lambda \mu}-\omega_{x} z_{\lambda \mu}\right]-\frac{B_{c}}{2} x_{\lambda \mu} \omega_{z}, \\
& p_{\lambda \mu}^{z}=\frac{\partial L}{\partial \dot{z}_{\lambda \mu}}=-\frac{B_{a}}{2} y_{\lambda \mu} \omega_{x}+\frac{B_{b}}{2} x_{\lambda \mu} \omega_{y}+B_{c}\left[\dot{z}_{\lambda \mu}+\omega_{x} y_{\lambda \mu}-\omega_{y} x_{\lambda \mu}\right] .
\end{aligned}
$$

Given the expressions of the canonical momenta $p_{\alpha}, p_{\beta}$, and $p_{\gamma}$, one obtains (by forming appropriate linear combinations) the components of angular momentum along the principal axes in the body-fixed frame:

$$
\begin{aligned}
& I_{1}=A_{a} \omega_{x}+\frac{B_{a}-2 B_{b}}{2} \sum_{\lambda \mu} \dot{y}_{\lambda \mu} z_{\lambda \mu}+\frac{2 B_{c}-B_{a}}{2} \sum_{\lambda \mu} y_{\lambda \mu} \dot{z}_{\lambda \mu} \\
& I_{2}=A_{b} \omega_{y}+\frac{B_{b}-2 B_{c}}{2} \sum_{\lambda \mu} \dot{z}_{\lambda \mu} x_{\lambda \mu}+\frac{2 B_{a}-B_{b}}{2} \sum_{\lambda \mu} z_{\lambda \mu} \dot{x}_{\lambda \mu} \\
& I_{3}=A_{c} \omega_{z}+\frac{B_{c}-2 B_{a}}{2} \sum_{\lambda \mu} \dot{x}_{\lambda \mu} y_{\lambda \mu}+\frac{2 B_{b}-B_{c}}{2} \sum_{\lambda \mu} x_{\lambda \mu} \dot{y}_{\lambda \mu} .
\end{aligned}
$$

One observes that both rotation and vibration contribute to the angular momentum. For the rotation, these are the usual products of moment of inertia and rotational frequency $\omega_{x}, \omega_{y}$, or $\omega_{z}$. For the vibration, the additional angular momentum arises from the motion of the nuclear surface with (anisotropic) mass parameters $B_{a, b, c}$.

In the expressions for $p_{\lambda \mu}^{x}, p_{\lambda \mu}^{y}$, and $p_{\lambda \mu}^{z}$, terms such as $\dot{x}_{\lambda \mu}$ are of order $\sqrt{\xi \Omega}$, while cross terms such as $\omega_{y} z_{\lambda \mu}$ are of order $\xi \sqrt{\xi / \Omega}$. Since the latter ones are suppressed by a factor $\xi / \Omega$, we can safely drop them. This leads to

$$
p_{\lambda \mu}^{x}=B_{a} \dot{x}_{\lambda \mu}, \quad p_{\lambda \mu}^{y}=B_{b} \dot{y}_{\lambda \mu}, \quad p_{\lambda \mu}^{z}=B_{c} \dot{z}_{\lambda \mu}
$$

such that the canonical momenta of vibrations are directly proportional to the velocities. 


\section{Effective Hamiltonian}

Applying the usual Legendre transformation, the effective Hamiltonian is obtained as

$$
\begin{aligned}
H= & \dot{\alpha} p_{\alpha}+\dot{\beta} p_{\beta}+\dot{\gamma} p_{\gamma}+\frac{1}{4 \pi} \int d \Omega\left(\dot{x} p^{x}+\dot{y} p^{y}+\dot{z} p^{z}\right)-L \\
= & \frac{A_{a}}{2} \omega_{x}^{2}+\frac{A_{b}}{2} \omega_{y}^{2}+\frac{A_{c}}{2} \omega_{z}^{2}+\frac{B_{a}}{2} \sum_{\lambda \mu} \dot{x}_{\lambda \mu}^{2}+\frac{B_{b}}{2} \sum_{\lambda \mu} \dot{y}_{\lambda \mu}^{2}+\frac{B_{c}}{2} \sum_{\lambda \mu} \dot{z}_{\lambda \mu}^{2}+\frac{1}{2} \sum_{\lambda \mu}\left\{\lambda(\lambda+1)\left[D_{a} x_{\lambda \mu}^{2}+D_{b} y_{\lambda \mu}^{2}+D_{c} z_{\lambda \mu}^{2}\right]\right. \\
& \left.+\mu^{2}\left[D_{d} x_{\lambda \mu}^{2}+D_{e} y_{\lambda \mu}^{2}+D_{f} z_{\lambda \mu}^{2}\right]\right\},
\end{aligned}
$$

with the rotational frequencies given by

$$
\begin{aligned}
& \omega_{x}=\frac{1}{A_{a}}\left[I_{1}-\frac{B_{a}-2 B_{b}}{2 B_{b}} \sum_{\lambda \mu} z_{\lambda \mu} p_{\lambda \mu}^{y}-\frac{2 B_{c}-B_{a}}{2 B_{c}} \sum_{\lambda \mu} y_{\lambda \mu} p_{\lambda \mu}^{z}\right], \\
& \omega_{y}=\frac{1}{A_{b}}\left[I_{2}-\frac{B_{b}-2 B_{c}}{2 B_{c}} \sum_{\lambda \mu} x_{\lambda \mu} p_{\lambda \mu}^{z}-\frac{2 B_{a}-B_{b}}{2 B_{a}} \sum_{\lambda \mu} z_{\lambda \mu} p_{\lambda \mu}^{x}\right], \\
& \omega_{z}=\frac{1}{A_{c}}\left[I_{3}-\frac{B_{c}-2 B_{a}}{2 B_{a}} \sum_{\lambda \mu} y_{\lambda \mu} p_{\lambda \mu}^{x}-\frac{2 B_{b}-B_{c}}{2 B_{b}} \sum_{\lambda \mu} x_{\lambda \mu} p_{\lambda \mu}^{y}\right],
\end{aligned}
$$

and the vibrational velocities are directly proportional to the momenta

$$
\dot{x}_{\lambda \mu}=\frac{p_{\lambda \mu}^{x}}{B_{a}}, \quad \dot{y}_{\lambda \mu}=\frac{p_{\lambda \mu}^{y}}{B_{b}}, \quad \dot{z}_{\lambda \mu}=\frac{p_{\lambda \mu}^{z}}{B_{c}}
$$

Substituting these relations into the Hamiltonian $H$, one extracts the leading-order part $(\sim \Omega)$ :

$$
H_{\Omega}=\sum_{\lambda \mu}\left\{\frac{\left(p_{\lambda \mu}^{x}\right)^{2}}{2 B_{a}}+\frac{\left(p_{\lambda \mu}^{y}\right)^{2}}{2 B_{b}}+\frac{\left(p_{\lambda \mu}^{z}\right)^{2}}{2 B_{c}}+\frac{1}{2} \lambda(\lambda+1)\left[D_{a} x_{\lambda \mu}^{2}+D_{b} y_{\lambda \mu}^{2}+D_{c} z_{\lambda \mu}^{2}\right]+\frac{1}{2} \mu^{2}\left[D_{d} x_{\lambda \mu}^{2}+D_{e} y_{\lambda \mu}^{2}+D_{f} z_{\lambda \mu}^{2}\right]\right\}
$$

This Hamiltonian describes a set of infinitely many uncoupled (anisotropic) harmonic oscillators with (vibrational) energies

$$
\begin{aligned}
& \Omega_{\lambda \mu}^{x}=\sqrt{\left[\lambda(\lambda+1) D_{a}+\mu^{2} D_{d}\right] / B_{a}}, \\
& \Omega_{\lambda \mu}^{y}=\sqrt{\left[\lambda(\lambda+1) D_{b}+\mu^{2} D_{e}\right] / B_{b}}, \\
& \Omega_{\lambda \mu}^{z}=\sqrt{\left[\lambda(\lambda+1) D_{c}+\mu^{2} D_{f}\right] / B_{c}},
\end{aligned}
$$

depending on the excitation mode $\lambda \mu$, the mass parameters $B_{a, b, c}$, and the parameters of the potential $D_{a-f}$. Correspondingly, the energy eigenvalues of this Hamiltonian are

$$
E_{\Omega}=\sum_{\lambda \mu}\left[\left(n_{\lambda \mu}^{x}+\frac{1}{2}\right) \Omega_{\lambda \mu}^{x}+\left(n_{\lambda \mu}^{y}+\frac{1}{2}\right) \Omega_{\lambda \mu}^{y}+\left(n_{\lambda \mu}^{z}+\frac{1}{2}\right) \Omega_{\lambda \mu}^{z}\right]
$$

with oscillator quantum numbers $n_{\lambda \mu}^{x, y, z}=0,1,2, \ldots$ and eigenfunctions given by products of Cartesian oscillator wave functions

$$
|\Phi\rangle=\prod_{\lambda \mu}\left|n_{\lambda \mu}^{x}\right\rangle\left|n_{\lambda \mu}^{y}\right\rangle\left|n_{\lambda \mu}^{z}\right\rangle .
$$

The parity of such a state is $(-1)^{\sum_{\lambda \mu}\left(n_{\lambda \mu}^{x}+n_{\lambda \mu}^{y}+n_{\lambda \mu}^{z}\right)}$, and since the ground state of an even-even nucleus has positive parity, one requires that $\sum_{\lambda \mu}\left(n_{\lambda \mu}^{x}+n_{\lambda \mu}^{y}+n_{\lambda \mu}^{z}\right)$ is even.

The NLO correction in the Hamiltonian $H$ is of order $\xi$. It takes the form of a rotational Hamiltonian

$$
H_{\xi}=\frac{\left(I_{1}-l_{1}\right)^{2}}{2 A_{a}}+\frac{\left(I_{2}-l_{2}\right)^{2}}{2 A_{b}}+\frac{\left(I_{3}-l_{3}\right)^{2}}{2 A_{c}},
$$


with the collective angular momenta shifted by vibrational contributions

$$
\begin{aligned}
& l_{1}=\frac{B_{a}-2 B_{b}}{2 B_{b}} \sum_{\lambda \mu} z_{\lambda \mu} p_{\lambda \mu}^{y}+\frac{2 B_{c}-B_{a}}{2 B_{c}} \sum_{\lambda \mu} y_{\lambda \mu} p_{\lambda \mu}^{z}, \\
& l_{2}=\frac{B_{b}-2 B_{c}}{2 B_{c}} \sum_{\lambda \mu} x_{\lambda \mu} p_{\lambda \mu}^{z}+\frac{2 B_{a}-B_{b}}{2 B_{a}} \sum_{\lambda \mu} z_{\lambda \mu} p_{\lambda \mu}^{x}, \\
& l_{3}=\frac{B_{c}-2 B_{a}}{2 B_{a}} \sum_{\lambda \mu} y_{\lambda \mu} p_{\lambda \mu}^{x}+\frac{2 B_{b}-B_{c}}{2 B_{b}} \sum_{\lambda \mu} x_{\lambda \mu} p_{\lambda \mu}^{y} .
\end{aligned}
$$

This implies that each vibrational state becomes a bandhead in the rotational spectrum.

As a side remark, we note that nuclei with axial symmetry are realized by the following parameters: $A_{b}=A_{a}, A_{c}=0, B_{b}=B_{a}$, $B_{c}=0, D_{b}=D_{a}, D_{c}=0, D_{e}=D_{d}$, and $D_{f}=0$. In this case, the $z_{\lambda \mu}$ degrees of freedom are absent, implying $l_{1}=l_{2}=0$. With the further constraint $I_{3}-l_{3}=0$ from $A_{c}=0$, the Hamiltonian $H=H_{\Omega}+H_{\xi}$ simplifies drastically to

$$
\begin{aligned}
H_{\Omega} & =\sum_{\lambda \mu}\left\{\frac{\left(p_{\lambda \mu}^{x}\right)^{2}+\left(p_{\lambda \mu}^{y}\right)^{2}}{2 B_{a}}+\frac{1}{2}\left[\lambda(\lambda+1) D_{a}+\mu^{2} D_{d}\right]\left[x_{\lambda \mu}^{2}+y_{\lambda \mu}^{2}\right]\right\}, \\
H_{\xi} & =\frac{I_{1}^{2}+I_{2}^{2}}{2 A_{a}}=\frac{I^{2}-I_{3}^{2}}{2 A_{a}},
\end{aligned}
$$

with

$$
I_{3}=B_{a} \sum_{\lambda \mu}\left(x_{\lambda \mu} \dot{y}_{\lambda \mu}-y_{\lambda \mu} \dot{x}_{\lambda \mu}\right)=\sum_{\lambda \mu}\left(x_{\lambda \mu} p_{\lambda \mu}^{y}-y_{\lambda \mu} p_{\lambda \mu}^{x}\right)
$$

consistent with the constructions in Refs. [12-14].

Moreover, if the vibrational degrees of freedom are neglected, the rotational Hamiltonian reduces to

$$
H_{\xi}=\frac{I_{1}^{2}}{2 A_{a}}+\frac{I_{2}^{2}}{2 A_{b}}+\frac{I_{3}^{2}}{2 A_{c}}
$$

as derived for the triaxial rotor at leading order in Ref. [20].

\section{PERTURBATIVE SOLUTION}

As mentioned above, the vibrations generate the contributions $l_{1,2,3}$ to the total angular momenta $I_{1,2,3}$. The rotor Hamiltonian $H_{\xi}$ in Eq. (76) involves infinitely many coupling terms, which makes it too complicated to be solved exactly. In the following, we use first-order perturbation theory to account for the vibrational corrections in $H_{\xi}$.

Let us start with the first term in $H_{\xi}$ proportional to

$$
\left(I_{1}-l_{1}\right)^{2}=I_{1}^{2}-2 I_{1} l_{1}+l_{1}^{2},
$$

and take its expectation value in a vibrational state $|\Phi\rangle$. Clearly, $I_{1}^{2}$ is not affected, since $|\Phi\rangle$ is independent of the Euler angles. The second term $I_{1} l_{1}$ gives zero, because the expectation value of a single position or momentum operator vanishes:

$$
\langle n|x| n\rangle=0, \quad\left\langle n\left|p^{x}\right| n\right\rangle=0 .
$$

Thus, we need to calculate only the expectation value of the third term,

$$
\begin{aligned}
l_{1}^{2}= & \left(\frac{B_{a}-2 B_{b}}{2 B_{b}}\right)^{2} \sum_{\lambda \mu} \sum_{\lambda^{\prime} \mu^{\prime}} z_{\lambda \mu} p_{\lambda \mu}^{y} z_{\lambda^{\prime} \mu^{\prime}} p_{\lambda^{\prime} \mu^{\prime}}^{y}+\left(\frac{2 B_{c}-B_{a}}{2 B_{c}}\right)^{2} \sum_{\lambda \mu} \sum_{\lambda^{\prime} \mu^{\prime}} y_{\lambda \mu} p_{\lambda \mu}^{z} y_{\lambda^{\prime} \mu^{\prime}} p_{\lambda^{\prime} \mu^{\prime}}^{z} \\
& +\left(\frac{B_{a}-2 B_{b}}{2 B_{b}}\right)\left(\frac{2 B_{c}-B_{a}}{2 B_{c}}\right) \sum_{\lambda \mu} \sum_{\lambda^{\prime} \mu^{\prime}}\left(z_{\lambda \mu} p_{\lambda^{\prime} \mu^{\prime}}^{z} p_{\lambda \mu}^{y} y_{\lambda^{\prime} \mu^{\prime}}+y_{\lambda \mu} p_{\lambda^{\prime} \mu^{\prime}}^{y} p_{\lambda \mu}^{z} z_{\lambda^{\prime} \mu^{\prime}}\right) .
\end{aligned}
$$

In the above double sums, the nondiagonal terms give still zero according to Eq. (85). For the diagonal terms

$$
\left(\frac{B_{a}-2 B_{b}}{2 B_{b}}\right)^{2} \sum_{\lambda \mu} z_{\lambda \mu}^{2} p_{\lambda \mu}^{y 2}+\left(\frac{2 B_{c}-B_{a}}{2 B_{c}}\right)^{2} \sum_{\lambda \mu} y_{\lambda \mu}^{2} p_{\lambda \mu}^{z 2}+\left(\frac{B_{a}-2 B_{b}}{2 B_{b}}\right)\left(\frac{2 B_{c}-B_{a}}{2 B_{c}}\right) \sum_{\lambda \mu}\left(z_{\lambda \mu} p_{\lambda \mu}^{z} p_{\lambda \mu}^{y} y_{\lambda \mu}+y_{\lambda \mu} p_{\lambda \mu}^{y} p_{\lambda \mu}^{z} z_{\lambda \mu}\right),
$$


the expectation values of squared positions, squared momenta, and products of position and momentum of the same kind in a harmonic oscillator state are easily computed. Altogether, the expectation value of $l_{1}^{2}$ in the state $|\Phi\rangle$ is given by

$$
\begin{aligned}
\left\langle l_{1}^{2}\right\rangle= & \frac{\left(B_{a}-2 B_{b}\right)^{2}}{16 B_{b} B_{c}} \sum_{\lambda \mu} \frac{\Omega_{\lambda \mu}^{y}}{\Omega_{\lambda \mu}^{z}}\left(2 n_{\lambda \mu}^{y}+1\right)\left(2 n_{\lambda \mu}^{z}+1\right) \hbar^{2}+\frac{\left(2 B_{c}-B_{a}\right)^{2}}{16 B_{b} B_{c}} \sum_{\lambda \mu} \frac{\Omega_{\lambda \mu}^{z}}{\Omega_{\lambda \mu}^{y}}\left(2 n_{\lambda \mu}^{z}+1\right)\left(2 n_{\lambda \mu}^{y}+1\right) \hbar^{2} \\
& +\frac{\left(B_{a}-2 B_{b}\right)\left(2 B_{c}-B_{a}\right)}{8 B_{b} B_{c}} \sum_{\lambda \mu} \hbar^{2} .
\end{aligned}
$$

It should be noted that this expression contains infinite sums that require in principle a regulator and eventual renormalization. In practice, one makes a truncation to the dominant lowest modes. The second and third terms in $H_{\xi}$ are treated in the same way. The inclusion of vibrational corrections to first order in the rotational Hamiltonian $H_{\xi}$ leads to the result

$$
\bar{H}_{\xi}=\frac{1}{2 A_{a}}\left[I_{1}^{2}+\sum_{\lambda \mu}\left(l_{\lambda \mu}^{1}\right)^{2}\right]+\frac{1}{2 A_{b}}\left[I_{2}^{2}+\sum_{\lambda \mu}\left(l_{\lambda \mu}^{2}\right)^{2}\right]+\frac{1}{2 A_{c}}\left[I_{3}^{2}+\sum_{\lambda \mu}\left(l_{\lambda \mu}^{3}\right)^{2}\right],
$$

with the mode-specific angular momentum shifts

$$
\begin{aligned}
& l_{\lambda \mu}^{1}=\left[\frac{\left(B_{a}-2 B_{b}\right)^{2}}{16 B_{b} B_{c}} \frac{\Omega_{\lambda \mu}^{y}}{\Omega_{\lambda \mu}^{z}}\left(2 n_{\lambda \mu}^{y}+1\right)\left(2 n_{\lambda \mu}^{z}+1\right)+\frac{\left(2 B_{c}-B_{a}\right)^{2}}{16 B_{b} B_{c}} \frac{\Omega_{\lambda \mu}^{z}}{\Omega_{\lambda \mu}^{y}}\left(2 n_{\lambda \mu}^{z}+1\right)\left(2 n_{\lambda \mu}^{y}+1\right)+\frac{\left(B_{a}-2 B_{b}\right)\left(2 B_{c}-B_{a}\right)}{8 B_{b} B_{c}}\right]^{1 / 2} \hbar, \\
& l_{\lambda \mu}^{2}=\left[\frac{\left(B_{b}-2 B_{c}\right)^{2}}{16 B_{c} B_{a}} \frac{\Omega_{\lambda \mu}^{z}}{\Omega_{\lambda \mu}^{x}}\left(2 n_{\lambda \mu}^{z}+1\right)\left(2 n_{\lambda \mu}^{x}+1\right)+\frac{\left(2 B_{a}-B_{b}\right)^{2}}{16 B_{c} B_{a}} \frac{\Omega_{\lambda \mu}^{x}}{\Omega_{\lambda \mu}^{z}}\left(2 n_{\lambda \mu}^{x}+1\right)\left(2 n_{\lambda \mu}^{z}+1\right)+\frac{\left(B_{b}-2 B_{c}\right)\left(2 B_{a}-B_{b}\right)}{8 B_{c} B_{a}}\right]^{1 / 2} \hbar, \\
& l_{\lambda \mu}^{3}=\left[\frac{\left(B_{c}-2 B_{a}\right)^{2}}{16 B_{a} B_{b}} \frac{\Omega_{\lambda \mu}^{x}}{\Omega_{\lambda \mu}^{y}}\left(2 n_{\lambda \mu}^{x}+1\right)\left(2 n_{\lambda \mu}^{y}+1\right)+\frac{\left(2 B_{b}-B_{c}\right)^{2}}{16 B_{a} B_{b}} \frac{\Omega_{\lambda \mu}^{y}}{\Omega_{\lambda \mu}^{x}}\left(2 n_{\lambda \mu}^{y}+1\right)\left(2 n_{\lambda \mu}^{x}+1\right)+\frac{\left(B_{c}-2 B_{a}\right)\left(2 B_{b}-B_{c}\right)}{8 B_{a} B_{b}}\right]^{1 / 2} \hbar .
\end{aligned}
$$

This shows that the angular momentum contributions from the vibrational motion play the role of recoil terms [18,19] in first-order perturbation theory. For different vibrational states, the spin components of the bandhead are different and characterized by the vibrational quantum numbers $n_{\lambda \mu}^{x}, n_{\lambda \mu}^{y}$, and $n_{\lambda \mu}^{z}$.

We can now specify the corrections for each vibrational state. For the ground state with quantum numbers $n_{\lambda \mu}^{x}=n_{\lambda \mu}^{y}=n_{\lambda \mu}^{z}=0$ and energy eigenvalue $E_{\Omega}=\frac{1}{2} \sum_{\lambda \mu}\left[\Omega_{\lambda \mu}^{x}+\Omega_{\lambda \mu}^{y}+\Omega_{\lambda \mu}^{z}\right]$, one has the angular momentum shifts

$$
\begin{aligned}
& l_{\lambda \mu}^{1}=\frac{\hbar}{4 \sqrt{B_{b} B_{c}}}\left|\left(B_{a}-2 B_{b}\right) \sqrt{\frac{\Omega_{\lambda \mu}^{y}}{\Omega_{\lambda \mu}^{z}}}+\left(2 B_{c}-B_{a}\right) \sqrt{\frac{\Omega_{\lambda \mu}^{z}}{\Omega_{\lambda \mu}^{y}}}\right|, \\
& l_{\lambda \mu}^{2}=\frac{\hbar}{4 \sqrt{B_{c} B_{a}}}\left|\left(B_{b}-2 B_{c}\right) \sqrt{\frac{\Omega_{\lambda \mu}^{z}}{\Omega_{\lambda \mu}^{x}}}+\left(2 B_{a}-B_{b}\right) \sqrt{\frac{\Omega_{\lambda \mu}^{x}}{\Omega_{\lambda \mu}^{z}}}\right|, \\
& l_{\lambda \mu}^{3}=\frac{\hbar}{4 \sqrt{B_{a} B_{b}}}\left|\left(B_{c}-2 B_{a}\right) \sqrt{\frac{\Omega_{\lambda \mu}^{x}}{\Omega_{\lambda \mu}^{y}}}+\left(2 B_{b}-B_{c}\right) \sqrt{\frac{\Omega_{\lambda \mu}^{y}}{\Omega_{\lambda \mu}^{x}}}\right| .
\end{aligned}
$$

Next, we consider the excitation of one particular mode $\lambda \mu$. Assuming the ordering of energies $\Omega_{\lambda \mu}^{x}<\Omega_{\lambda \mu}^{y}<\Omega_{\lambda \mu}^{z}$, the lowest vibrational excitation with positive parity [imposed by the parity-even unitary operator $U$ in Eq. (1)] has quantum numbers $n_{\lambda \mu}^{x}=2, n_{\lambda \mu}^{y}=n_{\lambda \mu}^{z}=0$. In this case, the excitation energy is $2 \Omega_{\lambda \mu}^{x}$ and the angular momentum shifts are

$$
\begin{aligned}
& l_{\lambda \mu}^{1}=\left[\frac{\left(B_{a}-2 B_{b}\right)^{2}}{16 B_{b} B_{c}} \frac{\Omega_{\lambda \mu}^{y}}{\Omega_{\lambda \mu}^{z}}+\frac{\left(2 B_{c}-B_{a}\right)^{2}}{16 B_{b} B_{c}} \frac{\Omega_{\lambda \mu}^{z}}{\Omega_{\lambda \mu}^{y}}+\frac{\left(B_{a}-2 B_{b}\right)\left(2 B_{c}-B_{a}\right)}{8 B_{b} B_{c}}\right]^{1 / 2} \hbar, \\
& l_{\lambda \mu}^{2}=\left[\frac{\left(B_{b}-2 B_{c}\right)^{2}}{16 B_{c} B_{a}} \frac{5 \Omega_{\lambda \mu}^{z}}{\Omega_{\lambda \mu}^{x}}+\frac{\left(2 B_{a}-B_{b}\right)^{2}}{16 B_{c} B_{a}} \frac{5 \Omega_{\lambda \mu}^{x}}{\Omega_{\lambda \mu}^{z}}+\frac{\left(B_{b}-2 B_{c}\right)\left(2 B_{a}-B_{b}\right)}{8 B_{c} B_{a}}\right]^{1 / 2} \hbar, \\
& l_{\lambda \mu}^{3}=\left[\frac{\left(B_{c}-2 B_{a}\right)^{2}}{16 B_{a} B_{b}} \frac{5 \Omega_{\lambda \mu}^{x}}{\Omega_{\lambda \mu}^{y}}+\frac{\left(2 B_{b}-B_{c}\right)^{2}}{16 B_{a} B_{b}} \frac{5 \Omega_{\lambda \mu}^{y}}{\Omega_{\lambda \mu}^{x}}+\frac{\left(B_{c}-2 B_{a}\right)\left(2 B_{b}-B_{c}\right)}{8 B_{a} B_{b}}\right]^{1 / 2} \hbar .
\end{aligned}
$$

One can see that, in comparison with that for the ground state band Eq. (91), the recoil terms are different in the excited states. 


\section{EXPRESSIONS IN TERMS OF QUADRUPOLE DEFORMATIONS}

In the construction of the effective Lagrangian (48), the vibrational degrees of freedom were denoted by $x, y$, and $z$. Its rotational part (proportional to $A_{a, b, c}$ ) involves vibrational contributions to the rotational frequencies and thus becomes rather complicated. Following experience, we express $x, y$, and $z$ in terms of the conventional quadrupole deformation parameters $\beta_{2}$ and $\gamma_{2}$ of a triaxially deformed nucleus.

Starting from the equation of the nuclear surface [19],

$$
\begin{aligned}
R(\theta, \phi)= & R_{0}\left\{1+\beta_{2}\left[\cos \gamma_{2} Y_{20}(\theta, \phi)\right.\right. \\
& \left.\left.+\frac{\sin \gamma_{2}}{\sqrt{2}}\left(Y_{22}(\theta, \phi)+Y_{2-2}(\theta, \phi)\right)\right]\right\},
\end{aligned}
$$

with $R_{0}=1.2 A^{1 / 3} \mathrm{fm}$, the displacements $x, y$, and $z$ take the form

$$
\begin{aligned}
x & =\left[R(\theta, \phi)-R_{0}\right] \sin \theta \cos \phi, \\
y & =\left[R(\theta, \phi)-R_{0}\right] \sin \theta \sin \phi, \\
z & =\left[R(\theta, \phi)-R_{0}\right] \cos \theta .
\end{aligned}
$$

By making use of the relations

$$
\begin{aligned}
\cos \theta Y_{\lambda \mu} & =a_{\lambda, \mu} Y_{\lambda+1, \mu}+a_{\lambda-1, \mu} Y_{\lambda-1, \mu}, \\
\sin \theta e^{i \phi} Y_{\lambda \mu} & =b_{\lambda-1,-(\mu+1)} Y_{\lambda-1, \mu+1}-b_{\lambda, \mu} Y_{\lambda+1, \mu+1}, \\
\sin \theta e^{-i \phi} Y_{\lambda \mu} & =-b_{\lambda-1, \mu-1} Y_{\lambda-1, \mu-1}+b_{\lambda,-\mu} Y_{\lambda+1, \mu-1},
\end{aligned}
$$

with the coefficients

$$
\begin{aligned}
& a_{\lambda \mu}=\sqrt{\frac{(\lambda+1)^{2}-\mu^{2}}{(2 \lambda+1)(2 \lambda+3)}} \\
& b_{\lambda \mu}=\sqrt{\frac{(\lambda+\mu+1)(\lambda+\mu+2)}{(2 \lambda+1)(2 \lambda+3)}},
\end{aligned}
$$

the few nonvanishing expansion coefficients $x_{\lambda \mu}, y_{\lambda \mu}$, and $z_{\lambda \mu}$ defined by Eq. (49) are given by

$$
\begin{aligned}
x_{31} & =-\sqrt{\frac{6}{35}} R_{0} \beta_{2} \cos \gamma_{2}+\sqrt{\frac{1}{70}} R_{0} \beta_{2} \sin \gamma_{2}, \\
x_{33} & =-\sqrt{\frac{3}{14}} R_{0} \beta_{2} \sin \gamma_{2}, \\
y_{3-1} & =-\sqrt{\frac{6}{35}} R_{0} \beta_{2} \cos \gamma_{2}-\sqrt{\frac{1}{70}} R_{0} \beta_{2} \sin \gamma_{2}, \\
y_{3-3} & =-\sqrt{\frac{3}{14}} R_{0} \beta_{2} \sin \gamma_{2}, \\
z_{30} & =\sqrt{\frac{9}{35}} R_{0} \beta_{2} \cos \gamma_{2}, \quad z_{32}=\sqrt{\frac{1}{7}} R_{0} \beta_{2} \sin \gamma_{2} .
\end{aligned}
$$

The vanishing of the coefficients with $\lambda=2$ has the following simple reason. The product $Y_{2 \mu} Y_{1 v}$ of spherical harmonics has odd parity, and therefore the parity-even functions $Y_{\lambda \mu^{\prime}}$ with $\lambda=2$ do not occur in its expansion in terms of spherical harmonics. With these restricted modes, the vibrational contributions to the angular momenta in Eqs. (61)-(63) actually vanish. With $l_{1,2,3}=0$, the rotational Hamiltonian reduces to that of a pure rotor, which can be solved exactly.

Substituting the modes (101)-(103) into the Lagrangian (51), calculating the corresponding canonical momenta, and performing the Legendre transformation, one arrives at the following LO Hamiltonian (vibrational part):

$$
H_{\Omega}=\frac{1}{2} B_{\beta \beta} \dot{\beta}_{2}^{2}+\frac{1}{2} B_{\gamma \gamma} \beta_{2}^{2} \dot{\gamma}_{2}^{2}+B_{\beta \gamma} \beta_{2} \dot{\beta}_{2} \dot{\gamma}_{2}+V,
$$

with the collective potential

$$
\begin{aligned}
V\left(\beta_{2}, \gamma_{2}\right)= & \frac{3}{35} R_{0}^{2}\left(12 D_{a}+12 D_{b}+18 D_{c}+D_{d}+D_{e}\right) \\
& \times \beta_{2}^{2} \cos ^{2} \gamma_{2}+\frac{2}{35} R_{0}^{2}\left(24 D_{a}+24 D_{b}+15 D_{c}\right. \\
& \left.+17 D_{d}+17 D_{e}+5 D_{f}\right) \beta_{2}^{2} \sin ^{2} \gamma_{2}+\frac{\sqrt{3}}{35} R_{0}^{2} \\
& \times\left(12 D_{b}+D_{e}-12 D_{a}-D_{d}\right) \beta_{2}^{2} \sin \gamma_{2} \cos \gamma_{2} .
\end{aligned}
$$

Furthermore, the mass parameters read

$$
\begin{aligned}
B_{\beta \beta}= & \frac{R_{0}^{2}}{35}\left[B_{a}\left(7-2 \sin \left(2 \gamma_{2}+\frac{\pi}{6}\right)\right)\right. \\
& \left.+B_{b}\left(7+2 \sin \left(2 \gamma_{2}-\frac{\pi}{6}\right)\right)+7 B_{c}\left(1+\cos 2 \gamma_{2}\right)\right], \\
B_{\gamma \gamma}= & \frac{R_{0}^{2}}{35}\left[B_{a}\left(7+2 \sin \left(2 \gamma_{2}+\frac{\pi}{6}\right)\right)\right. \\
& \left.+B_{b}\left(7-2 \sin \left(2 \gamma_{2}-\frac{\pi}{6}\right)\right)+7 B_{c}\left(1-\cos 2 \gamma_{2}\right)\right], \\
B_{\beta \gamma}= & \frac{R_{0}^{2}}{35}\left[2 B_{a} \sin \left(2 \gamma_{2}-\frac{\pi}{3}\right)+2 B_{b} \sin \left(2 \gamma_{2}+\frac{\pi}{3}\right)\right. \\
& \left.-2 B_{c} \sin 2 \gamma_{2}\right],
\end{aligned}
$$

and, interestingly, they do not depend on $\beta_{2}$.

Moreover, the rotational part of the NLO Hamiltonian is just

$$
H_{\xi}=\frac{I_{1}^{2}}{2 A_{a}}+\frac{I_{2}^{2}}{2 A_{b}}+\frac{I_{3}^{2}}{2 A_{c}},
$$

with moments of inertia $A_{a}, A_{b}$, and $A_{c}$ independent of the deformation parameters $\beta_{2}$ and $\gamma_{2}$.

The total Hamiltonian

$$
H=H_{\Omega}+H_{\xi}
$$

is a rotation-vibration Hamiltonian of the Bohr-Mottelson type [18] with an explicit potential $V$ and nonconstant mass parameters. In the following, this Hamiltonian is used to describe the experimental energy spectra.

\section{RESULTS AND DISCUSSIONS}

In this section, the experimental ground state bands, $\gamma$ bands, and $K=4$ bands for the isotopes ${ }^{108,110,112} \mathrm{Ru}$ are 


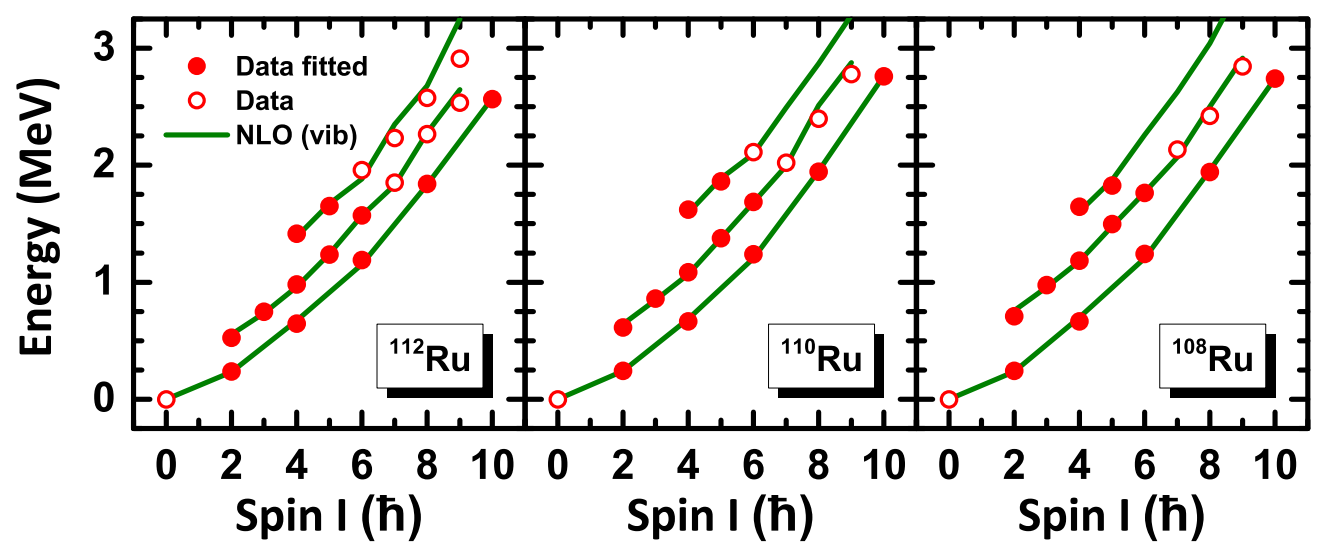

FIG. 1. Energy spectra for the ground state, $\gamma$, and $K=4$ bands in ${ }^{108-112} \mathrm{Ru}$ calculated by rotation-vibration Hamiltonian (108).

used to examine the applicability of the present EFT in the description of collective rotations and vibrations of triaxially deformed nuclei. The data are taken from the compilation of the National Nuclear Data Center (NNDC) [28]. In Ref. [20], it was shown that these three $\mathrm{Ru}$ isotopes have a triaxially deformed minimum and exhibit softness along the $\gamma_{2}$ direction in the potential energy surface, as calculated by CDFT [25]. Moreover, the breakdown scale of the EFT marks the appearance of neglected degrees of freedom, e.g., single-particle degrees of freedom or pair-breaking effects. This value is about $2-3 \mathrm{MeV}$, which corresponds to the excitation energy of the state with $I=10 \hbar$. In Ref. [20], the nearly constant behavior of the experimental alignments in the spin region of $I \leqslant 10 \hbar$ was shown, which indicates that the corresponding data are not beyond the breakdown energy scale for collective rotational and vibrational motions (i.e., beyond the pairing instability). Therefore, the application of the rotation-vibration Hamiltonian (108) is restricted to this spin region.

In Fig. 1, the energy spectra of the ground state bands, $\gamma$ bands, and $K=4$ bands obtained from rotation-vibration Hamiltonian (108) are shown as a function of spin $I$ for the isotopes ${ }^{108,110,112} \mathrm{Ru}$, respectively. The parameters of the Hamiltonian are obtained by fitting to the solid data points in Fig. 1, and their values are listed in Table I. One can see that the data are well reproduced by the rotation-vibration Hamiltonian (108). In particular, the obvious staggering behavior of the $\gamma$ bands found with the pure triaxial rotor Hamiltonian [20] is no longer present. This improved description underlines the importance of including vibrational degrees of freedom in the EFT formulation. In the present EFT, the LO terms scale as $\Omega$, the NLO terms as $\Omega(\xi / \Omega)$, and the neglected higher-order terms are at least of order $\Omega(\xi / \Omega)^{2}$. In the Ru isotopes, $\Omega \approx 0.75 \mathrm{MeV}, \xi \approx 0.25 \mathrm{MeV}$, and thus $\Omega(\xi / \Omega)^{2}$ is about $0.08 \mathrm{MeV}$. This can be taken as a qualitative uncertainty estimate.

From the moments of inertia, mass parameters, and potential parameters collected in Table I, one recognizes significant differences between the isotopes ${ }^{108} \mathrm{Ru}$ and ${ }^{112,110} \mathrm{Ru}$. This is consistent with the fact that the energy differences between the ground state and $\gamma$ bands in ${ }^{108} \mathrm{Ru}(\sim 500 \mathrm{keV})$ are larger than those in ${ }^{112,110} \mathrm{Ru}(\sim 350 \mathrm{keV})$. This also indicates that one has to fit the parameters for each nucleus separately, and this, to some extent, weakens the predictive power of the EFT.

Let us discuss the fitted values of parameters collected in Table I. From the lowest $2_{1}^{+}$state at $0.25 \mathrm{MeV}$, one estimates $A_{i} \sim 24 \hbar^{2} / \mathrm{MeV}$, which sets the natural scale. Table I now reveals that $B_{a}$ and $B_{b}$ are of similar size, while the fitted central value of $B_{c}$ comes out unnaturally small for all three $\mathrm{Ru}$ isotopes. It is, however, accompanied by a sizeable error, comparable to that of the other parameters. The parameters $D_{i}$, scaling as $\Omega / \xi^{2}$, are mostly of natural size. A more detailed statistical analysis of the fitting procedure is needed to judge whether all parameters are of natural size.

With the parameters listed in Table I, the collective potential $V\left(\beta_{2}, \gamma_{2}\right)$ [Eq. (105)] and the mass parameters $B_{\beta \beta}, B_{\beta \gamma}$, and $B_{\gamma \gamma}$ [Eqs. (106)] are determined and these are shown for the three Ru isotopes in Figs. 2 and 3, respectively. One observes that the potential energy surfaces shown in Fig. 2 possess similar shapes. Namely, there is a spherical minimum and softness along the $\beta_{2}$ direction with a valley located around $\gamma_{2}=20^{\circ}$. It should be noted that such a behavior of the potential energy surface is different from those calculated by CDFT [20,25] using the effective interaction PC-PK1 [29], in which a triaxially deformed minimum and softness along the

TABLE I. Parameters used in the rotation-vibration Hamiltonian (108) for calculations of ${ }^{108-112} \mathrm{Ru}$. The units of $A_{a, b, c}$ are $\hbar^{2} / \mathrm{MeV}$, the units of $B_{a, b, c}$ are $\hbar^{2} /\left(\mathrm{MeV} \mathrm{fm}^{2}\right)$, and the units of $D_{a-f}$ are $\mathrm{MeV} / \mathrm{fm}^{2}$.

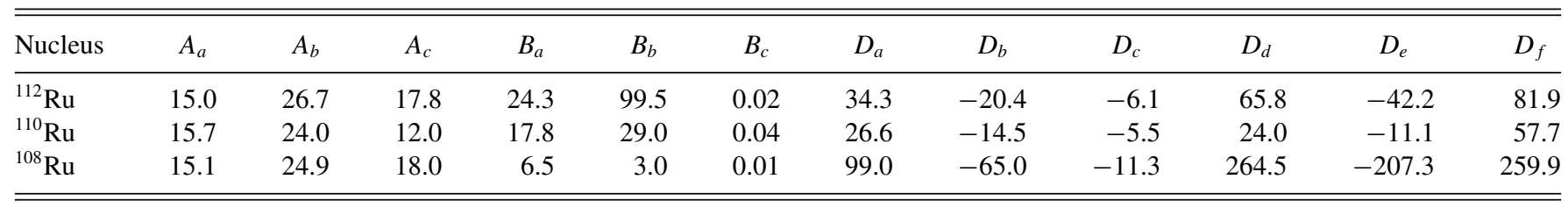



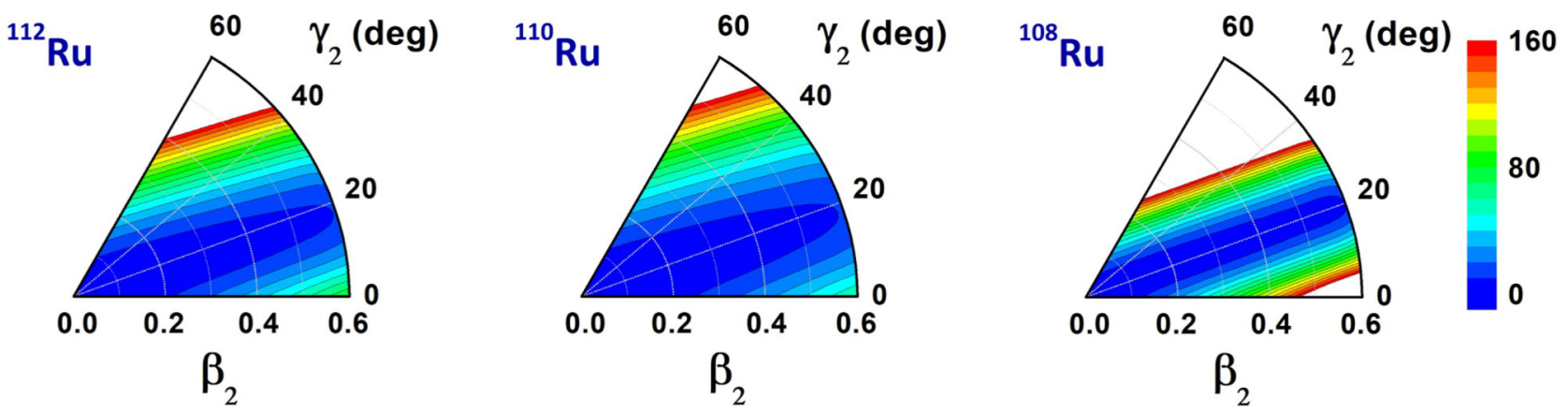

FIG. 2. Collective potentials for ${ }^{108-112}$ Ru calculated by Eq. (105).

$\gamma_{2}$ direction are found. The difference is due to the fact that only terms proportional to $\beta_{2}^{2}$ are present in the $\mathrm{LO}$ collective potential (105). In order to get a more structured shape of the potential energy surface of CDFT, the higher-order terms $\mathcal{L}_{5 i}$ and $\mathcal{L}_{6 i}$ in Eq. (48) need to be kept. The other difference from CDFT is that the moments of inertia are constants for the different deformations $\left(\beta_{2}, \gamma_{2}\right)$ in the EFT formulation. This also indicates that the EFT formulation gives a different picture for the descriptions of the energy spectra of $\mathrm{Ru}$ isotopes in comparison to the 5DCH based on CDFT [20].

We have already mentioned that the mass parameters $B_{\beta \beta}$, $B_{\beta \gamma}$, and $B_{\gamma \gamma}$ are independent of the deformation $\beta_{2}$. Furthermore, their dependence on $\gamma_{2}$ is moderate, as can be seen in Fig. 3. With increasing $\gamma_{2}, B_{\beta \beta}$ increases and $B_{\gamma \gamma}$ decreases. One can observe that $B_{\beta \gamma}$ is much smaller than $B_{\beta \beta}$ and $B_{\gamma \gamma}$ for all three Ru isotopes, which implies that the coupling between $\beta_{2}$ and $\gamma_{2}$ is small. Once again, we should point out that the EFT formulation gives a different picture for collective energy spectra in comparison to those of the 5DCH based on CDFT [20].

Here, we would like to add some remarks about the differences between the (covariant) density functional theory and EFT in the descriptions of the low-lying states. In the (covariant) density functional theory, no adjustable parameters are needed once the functional is determined, and it can be used for describing almost all of the nuclei in the nuclear chart. From it, the moments of inertia, mass parameters, and the collective potential can be obtained and fed into a collective Hamiltonian to describe the low-lying states. In an EFT for collective motion, one has to fit the parameters for each nucleus separately. This weakens the predictive power of the EFT to some extent. However, one can address easily and unambiguously the uncertainties of the description for the data in the EFT as one expands it order by order and, hence, can give a high-precision description for the experimental data.

\section{SUMMARY}

In summary, the EFT for triaxially deformed even-even nuclei was generalized to include the vibrational degrees of freedom. The pertinent Lagrangian and Hamiltonian were obtained up to NLO. The LO Hamiltonian describes a set of uncoupled (anisotropic) harmonic oscillators. The NLO part couples rotations to vibrations, and it is found that the vibrations provide contributions to the angular momenta $I_{1}$, $I_{2}$, and $I_{3}$. This coupling makes the rotational Hamiltonian too complicated to be solved exactly.

Therefore, we have treated the NLO (rotational) Hamiltonian in first-order perturbation theory. This leads to corrections from the vibrational motion in the form of so-called recoil terms. For different vibrational states, the spin components of the bandhead become different and they depend on the vibrational quantum numbers.

The NLO Hamiltonian has also been expressed in terms of quadrupole deformation parameters $\beta_{2}$ and $\gamma_{2}$. A

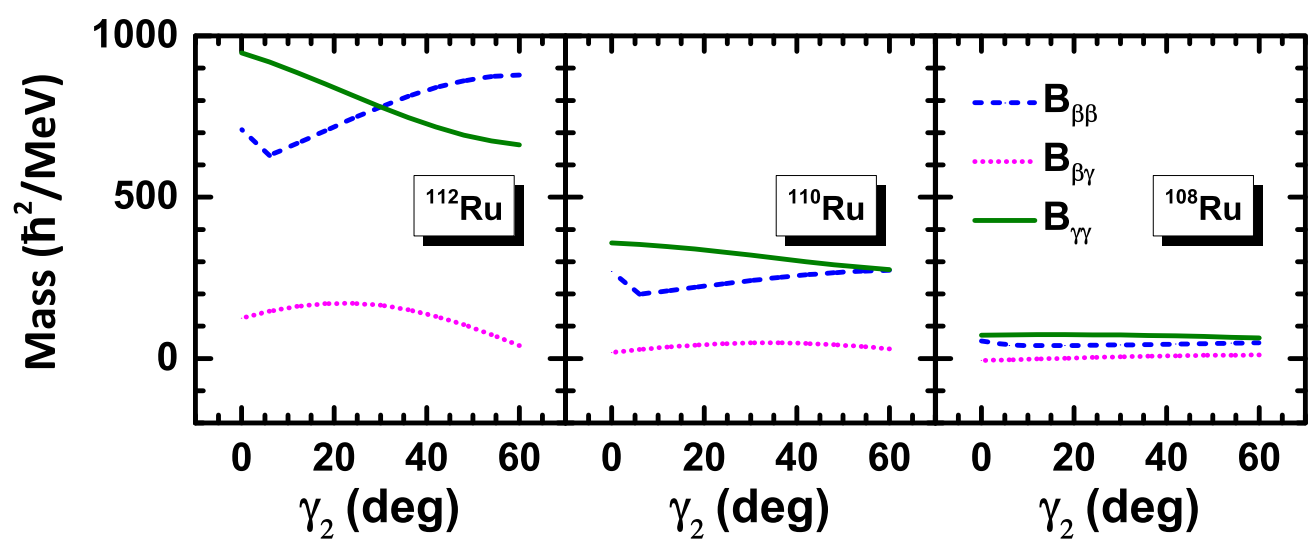

FIG. 3. Mass parameters for ${ }^{108-112}$ Ru calculated by Eq. (106). 
rotation-vibration Hamiltonian (without mutual coupling) is obtained. Its applicability was examined in the description of the energy spectra of the ground state bands, $\gamma$ bands, and $K=4$ bands in ${ }^{108,110,112} \mathrm{Ru}$ isotopes. It is found that, by taking into account the vibrational degree of freedom, the deviations for high-spin states in the $\gamma$ band, using the EFT with only rotational degree of freedom, disappear. This underlines the importance of including vibrational degrees of freedom in the EFT formulation.

The results presented in this work give us confidence to further generalize the EFT for triaxially deformed nuclei with odd mass number, which requires a systematic treatment of the coupling between the single-particle motion and the collective rotational motion [30].

\section{ACKNOWLEDGMENTS}

The authors thank T. Papenbrock, P. Ring, and W. Weise for helpful and informative discussions. This work was supported in part by the Deutsche Forschungsgemeinschaft (DFG) and National Natural Science Foundation of China (NSFC) through funds provided to the Sino-German CRC 110 "Symmetries and the Emergence of Structure in QCD," the National Key R\&D Program of China (Contract No. 2018YFA0404400) and the NSFC under Grants No. 11335002 and No. 11621131001. The work of U.G.M. was also supported by the Chinese Academy of Sciences (CAS) President's International Fellowship Initiative (PIFI) (Grant No. 2018DM0034) and by VolkswagenStiftung (Grant No. 93562).
[1] E. Epelbaum, Prog. Part. Nucl. Phys. 57, 654 (2006).

[2] E. Epelbaum, H.-W. Hammer, and U.-G. Meißner, Rev. Mod. Phys. 81, 1773 (2009).

[3] R. Machleidt and D. R. Entem, Phys. Rep. 503, 1 (2011).

[4] C. Bertulani, H.-W. Hammer, and U. van Kolck, Nucl. Phys. A 712, 37 (2002).

[5] H.-W. Hammer and D. Phillips, Nucl. Phys. A 865, 17 (2011).

[6] E. Ryberg, C. Forssén, H.-W. Hammer, and L. Platter, Phys. Rev. C 89, 014325 (2014).

[7] P. F. Bedaque and U. van Kolck, Annu. Rev. Nucl. Part. Sci. 52, 339 (2002).

[8] H. Grießhammer, J. McGovern, D. Phillips, and G. Feldman, Prog. Part. Nucl. Phys. 67, 841 (2012).

[9] H.-W. Hammer, A. Nogga, and A. Schwenk, Rev. Mod. Phys. 85, 197 (2013).

[10] T. Papenbrock, Nucl. Phys. A 852, 36 (2011).

[11] J. L. Zhang and T. Papenbrock, Phys. Rev. C 87, 034323 (2013).

[12] T. Papenbrock and H. A. Weidenmüller, Phys. Rev. C 89, 014334 (2014).

[13] T. Papenbrock and H. A. Weidenmüller, J. Phys. G: Nucl. Part. Phys. 42, 105103 (2015).

[14] T. Papenbrock and H. A. Weidenmüller, Phys. Scr. 91, 053004 (2016).

[15] E. A. Coello Pérez and T. Papenbrock, Phys. Rev. C 92, 014323 (2015).
[16] E. A. Coello Pérez and T. Papenbrock, Phys. Rev. C 92, 064309 (2015).

[17] E. A. Coello Pérez and T. Papenbrock, Phys. Rev. C 94, 054316 (2016).

[18] A. Bohr and B. R. Mottelson, Nuclear Structure (Benjamin, New York, 1975), Vol. II.

[19] P. Ring and P. Schuck, The Nuclear Many Body Problem (Springer-Verlag, Berlin, 1980).

[20] Q. B. Chen, N. Kaiser, U.-G. Meißner, and J. Meng, Eur. Phys. J. A 53, 204 (2017).

[21] R. Bengtsson, H. Frisk, F. May, and J. Pinston, Nucl. Phys. A 415, 189 (1984).

[22] I. Hamamoto and H. Sagawa, Phys. Lett. B 201, 415 (1988).

[23] S. Frauendorf and J. Meng, Nucl. Phys. A 617, 131 (1997).

[24] J. Meng, J. Peng, S. Q. Zhang, and S.-G. Zhou, Phys. Rev. C 73, 037303 (2006).

[25] Relativistic Density Functional for Nuclear Structure, edited by J. Meng, International Review of Nuclear Physics Vol. 10 (World Scientific, Singapore, 2016).

[26] S. Coleman, J. Wess, and B. Zumino, Phys. Rev. 177, 2239 (1969).

[27] C. G. Callan, S. Coleman, J. Wess, and B. Zumino, Phys. Rev. 177, 2247 (1969).

[28] http://www.nndc.bnl.gov/ensdf/

[29] P. W. Zhao, Z. P. Li, J. M. Yao, and J. Meng, Phys. Rev. C 82, 054319 (2010).

[30] E. Streck, Q. B. Chen, N. Kaiser, and Ulf-G. Meißner, arXiv:1805.03633. 